\title{
A clearer spotlight on spotlight: Understanding, conducting and reporting 2 s
}

\author{
Aradhna Krishna \\ Ross School of Business, University of Michigan, 701 Tappan St., Ann Arbor, MI 48109-1234,USA
}

Accepted by Amna Kirmani, Editor

Received 6 April 2016; accepted 8 April 2016

Available online 28 April 2016

\begin{abstract}
There has been a remarkable increase in the use of spotlight analysis to examine any interactive effect between an independent variable and a continuous moderator. Most of the spotlight analyses have been conducted at one standard deviation above and below the mean value of the moderator, even when alternate methods are more appropriate. Additionally, many spotlight analyses are not conducted correctly. More importantly, results for spotlight analyses are reported in a manner that makes it virtually impossible for mistakes to be detected. This article focuses on "understanding," "conducting," and "reporting" spotlight analyses. By posing questions for the reader, it highlights some common mistakes made when doing spotlight analysis and explains why confusion often arises. Then it provides an easy to understand way to do spotlight analysis for some popular contexts. Alternatives to spotlight analysis are also briefly discussed. Finally, it suggests how to report results for spotlight analysis and for the alternatives. Pointing out recurrent mistakes should prevent perpetuation of misleading practices. Similarly, reporting essential details of the analyses should prevent mistakes from going undetected.
\end{abstract}

(C) 2016 Society for Consumer Psychology. Published by Elsevier Inc. All rights reserved.

Keywords: Spotlight analysis; Johnson-Neyman technique; Dichotomizing variables

The nature of consumer psychology research is such that we often want to examine the effect of one variable at certain levels of another variable. Frequently, one of these variables is continuous in nature - for instance, demographic variables like age, weight, height; product features like price, fuel efficiency, volume; marketing variables like advertising dollars spent, promotion dollars spent, et cetera. There are also variables that are measured on constructed continuous scales such as self-control, need-for-touch, style of processing, vividness of visual imagery, and self-esteem.

The consumer psychologist frequently wants to study the effect of two variables, one of which is continuous, on a dependent

it This article is based on many discussions I have had with my doctoral students and post-docs and participants in my Sensory Marketing Laboratory (www.sensorymarketinglab.com), especially Luca Cian and Ryan Elder. I would also like to thank Nilufer Aydinoglu, Fred Feinberg, Amna Kirmani, Maureen Morrin, Tatiana Sokolova, and three pairs of anonymous reviewers for comments on this article.

E-mail address: aradhna@umich.edu. variable. For instance, she may want to study if touching or not a given food ("Touch-food"-yes, no) affects its "Consumption" (the amount of the food that is eaten). She may also want to look at the effect of "self-control" on such "consumption" (see Fig. 1 for a hypothetical Effect of Consumer Self-control and Touching Food on Consumption). If she finds a significant interactive effect between the two variables, it implies that the effect of one variable, Touch-food (the independent variable), is dependent on the values of the other variable, Self-control (the moderator). In this case, she may want to examine the effect of Touch-food at certain levels of the continuous variable, Self-control. What should she do then? The way in which consumer researchers have further explored such interaction effects between a categorical independent variable and a continuous independent variable has varied over time.

Until about a decade ago, it used to be the case that researchers did a median split on the moderator, Self-control, effectively turning the continuous variable into a binary variable with two values-high Self-control and low Self-control. Then they 


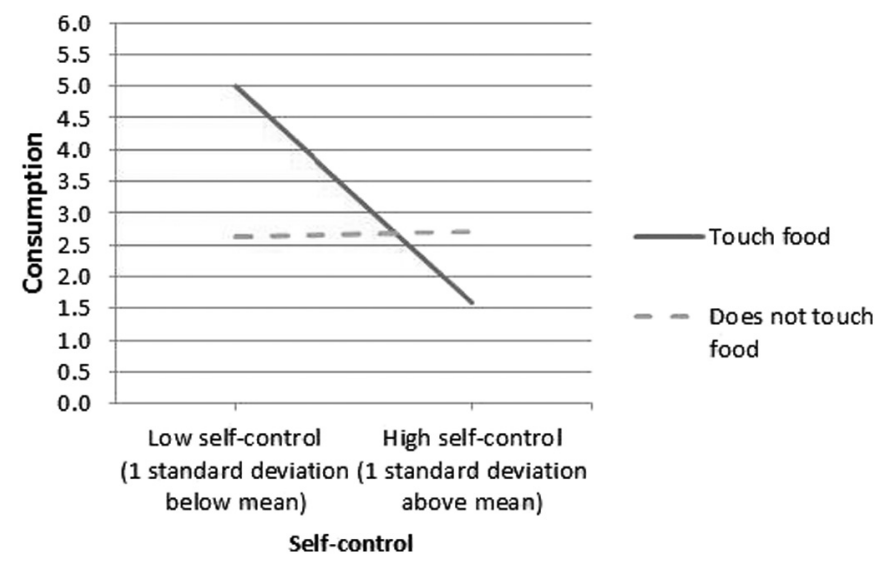

Fig. 1. Hypothetical effect of self-control and touch-food (yes/not) on consumption (self-control at 1 standard deviation above/below the mean).

examined if the effect of the independent variable, Touch-food, depended on the value of the binary moderator, typically using simple effect tests (which were often called contrast tests). Hence, one would analyze the data to see if Touch-food had a significant effect on Consumption within low values of Self-control, and also within high values of Self-control. One reason for this dichotomy of the continuous variable into a high and a low value was researchers' belief that this would ease the explanation of any interactive effect between an independent variable and moderator.

Unfortunately, past literature has shown that analyses based on mean/median splits on the continuous moderator can give both false positive and false negative results (Irwin \& McClelland, 2003). Also, dichotomizing the continuous moderator treats responses at very small and very large distances from the split to be the same - thus, 1 versus 7 on a 7-point scale would be treated the same as a response of 3.99 and 4.01 if the split happened at 4 . This swallows some of the statistical power from the analysis, reducing the ability to diagnose a significant interaction.

This problem was realized by a number of researchers in the consumer psychology and the broader psychology community and a number of articles on this issue were written. For a recent dialogue on when to dichotomize variables, see Pham (2015); Iacobucci, Posovac, Kardes, Schneider, and Popovich (2015a, 2015b); Rucker, McShane, and Preacher (2015); McClelland, Lynch, Irwin, Spiller, and Fitzsimons (2015). Early discussions on the subject were provided by Jaccard, Turrisi, and Wan (1990); Aiken and West (1991); and Cohen (1983), and followed up by Irwin and McClelland (2001, 2003) and MacCallum, Zhang, Preacher, and Rucker (2002). Jaccard et al. (1990), Aiken and West (1991), and Irwin and McClelland (2001) proposed an alternate approach where one looked at the effect of the independent variable at low and high values of the moderator by mean shifting the data (and not by doing a median split on the data); Irwin and McClelland (2001) called this "pointing a spotlight on the model from different angles". Fitzsimons (2008) subsequently wrote a short editorial based on these longer articles dubbing the proposed alternative approach "spotlight analysis."

Fitzsimons (2008) provided an illustrative spotlight analysis which suggested looking at the effect of the independent variable at one standard deviation above and below the mean value of the moderator. This illustration proved to be very persuasive. Since then, dozens of spotlight analyses have been done, and most of them have been conducted at those two suggested values of the moderator. In the Journal of Consumer Research, 1, 4, 3, 12, 10, 18, 24, and 17 papers used a spotlight analysis in 2008, 2009, 2010, 2011, 2012, 2013, 2014, and 2015, respectively. The count for the Journal of Marketing Research in the same years is $1,1,0,4,4,7,2$, and 2, and for the Journal of Consumer Psychology, it is 0, 0, 2, 1, 3, 5, 7, and 10. It appears that it has become de rigueur to use spotlight analysis for analyzing an interactive effect.

Unfortunately, three problems have arisen with the current way in which spotlight analysis is being done. First, while Fitzsimons (2008) suggested using spotlight analysis instead of dichotomizing continuous variables, he did not exactly spell out in any easy way how to do it. Jaccard et al. (1990); Aiken and West (1991) and Irwin and McClelland (2001) give more detail but either researchers find their articles hard to comprehend or do not take the effort to understand them. In any event, it appears that researchers have since struggled, often doing the analysis wrong. Thus, there are problems in understanding and conducting spotlight analysis correctly. Second and more disturbing is the fact that the method for reporting spotlight analyses (which often follows Fitzsimons, 2008) makes such errors impossible to detect. Spiller, Fitzsimons, Lynch, and McClelland (2013) report that in volume 48 of the Journal of Marketing Research and volume 38 of the Journal of Consumer Research, many of the analyses were not optimal and others were simply wrong. Publishing analyses that were incorrect could be prevented by better understanding and reporting of analyses. Lastly, there are other methods for doing spotlight analyses, aside from examining the effect of the moderator at 1 standard deviation above and below the mean, and these are more appropriate in many cases.

The tutorial of Spiller et al. (2013) is a start to focus on the current spotlight analysis problems. The authors concentrate on the third and last of the issues mentioned above - alternative ways of conducting spotlight analyses. The authors' hope is that their tutorial will reduce spotlight analyses being done at one standard deviation above and below the mean, and that focal points and the JohnsonNeyman technique will be used instead in many instances. They clarify how to do spotlight analyses at focal points using several different experimental designs and when to choose the Johnson-Neyman technique (which they term "floodlight analyses") over spotlight analyses.

This article continues the focus on the current problems with spotlight analysis. However, in contrast to Spiller et al. (2013), it lays more emphasis on the first two problems, "understanding and conducting," and "reporting," compared to the third (alternative methods to do spotlight analysis). As such, the article (i) highlights some common incorrect ways in which spotlight analysis is currently done, which also underscores the puzzling nature of spotlight analysis. In order to do this, the article poses several multiple choice questions to the reader. Then it provides the correct solution to these questions and explains why the correct solution is right. The 
article also (ii) offers an intuitive understanding of a few common contexts where spotlight analysis is typically done along with simple ways in which to conduct these analyses. Alternative analyses to spotlight analysis are also discussed in brief. Lastly, it (iii) explains exactly how to report the results for spotlight analysis and its alternatives, so that the method used by the researchers is transparent. This will prevent mistakes from being hidden and never discovered; and therefore prevent erroneous conclusions.

My belief is that unless the common incorrect ways to do spotlight analysis are highlighted and simple intuitive steps for the correct way to do the analysis provided, mistakes may continue to be made. I also fear that reporting may remain opaque, covering any mistakes that have been made. As such, I hope that this article will prove to be insightful to the consumer psychology community.

\section{Understanding spotlight analysis: highlighting common confusion}

To show that researchers are struggling with the correct way to do spotlight analyses, I present a scenario and pose four questions. If you struggle with the answer, this article is indeed useful. If you do not struggle, but get the answer wrong, the article is even more useful.

Let's reconsider the example given earlier. A consumer psychologist is studying the effect of being able to Touch-food (yes versus no) and Self-control (a measured variable) on Consumption. She chooses to use dummy coding for Touch-food (Touch-food $=1$ when one can touch food and 0 when one cannot) and estimates the following model:

$$
\begin{aligned}
\text { Consumption }= & a+b \text { Self }- \text { control }+c \text { Touch }- \text { food } \\
& +d(\text { Self }- \text { control } \times \text { Touch }- \text { food })
\end{aligned}
$$

She gets a significant interaction between Self-control and Touch-food and now wants to perform a spotlight analysis to see the effect of Touch-food at high levels of Self-control.

Since many researchers have been using Fitzsimons's (2008) paper to report their analysis and the reader needs to be able to understand what the researchers have done, I cite Fitzsimons here (the paraphrasing changes the Fitzsimons' example to the one I used earlier):

"... the researcher performs a "spotlight" analysis at one or more standard deviations above the mean of Self-control. By mean-shifting the Self-control data up or down (i.e., adding a constant to all Self-control responses, thus "shifting" the mean higher or lower) the researcher can focus the "spotlight" on the region of Self-control in which the researcher would like to test for differences across [Touchfood yes or no] conditions. At, for example, one standard deviation above the mean level of Self-control, the statistical test for differences across [Touch-food yes or no] is given by the parameter and significance of the Touch-food yes dummy variable in the regression equation..."
Please answer the following questions (choose one of the options):

Question 1: The puzzling nature of mean shifts (conducting spotlight-categorical IV and continuous moderator). What should a researcher do to run the spotlight analysis correctly if she estimates equation (1) and finds a significant interaction between Touch-food and Self-control (i.e., $d$ is significant)? Note that Touch-food is a categorical variable (yes/no) and Self-control is continuous [note also that in all illustrations in the paper, the continuous variable is the raw data, neither mean-centered nor standardized]. She wants to examine the effect of Touch-food at high levels of Self-control, i.e., wants to do a spotlight analysis at high levels of Self-control. As stated above, she would mean shift the data "up" by 1 standard deviation of Self-control. Say the mean of Self-control $=m$ and the standard deviation $=s$. What is the correct way to do this mean shift?

Option 1: In the data, she replaces Self-control with Self-control ${ }^{\prime}=$ Self-control $-m+s$

Option 2: In the data, she replaces Self-control with Self-control ${ }^{\prime}=$ Self-control $-m-s$

Option 3: In the data, she replaces Self-control with Self-control ${ }^{\prime}=$ Self-control $+s$

Option 4: In the data, she replaces Self-control with Self-control' $=$ Self-control $-s$

Option 5: Other

Answer for Question 1: The correct answer is option 2. In her data, the researcher should replace Self-control by Self-control $^{\prime}=$ Self-control $-m-s$. Then she should estimate equation (2) below. Equation (2) is simply equation (1) with all instances of Self-control replaced with Self-control'

$$
\begin{aligned}
\text { Consumption }= & a+b \text { Self }- \text { control }^{\prime}+c \text { Touch }- \text { food } \\
& +d\left(\text { Self }- \text { control }^{\prime} \times \text { Touch }- \text { food }\right)
\end{aligned}
$$

The correct answer of option 2 may seem counterintuitive and puzzling - in Q1, why are we "subtracting" the mean and standard deviation when we are supposedly mean shifting the data "up" and trying to reflect a "high" level of Self-control? The reason is as follows: when Self-control" $=0$, then equation (2) reduces to Consumption $=a+c$ Touch-food. If $c$ is significant in this equation, then it implies that Touch-food affects Consumption when Self-control' $=0$. However, how does this capture the effect of Touch-food on consumption at high self-control? Remember that when Self-control ${ }^{\prime}=0$, the original raw score for Self-control $=m+s$, which is the high level of Self-control (1 standard deviation above the mean), and what we want to focus on. Basically, the main idea here is to use a linear transformation of the variable in question to set the specific value of interest to zero.

Question 2: What is the output of interest (interpreting spotlight_categorical IV and continuous moderator)? Let's say the researcher mean shifts the data and runs the new regression for Question 1 (i.e., instead of estimating equation (1), she estimates equation (2)). To see if the effect of Touch-food at 
high levels of Self-control is significant, she should look at the following:

Option 1: whether $b$ is significant

Option 2: whether $c$ is significant

Option 3: whether $d$ is significant

Answer for Question 2: After estimating equation (2), to see if the effect of Touch-food at high levels of Self-control is significant, we need to look at whether $c$ is significant (i.e., the correct answer is option 2). Again, note that we want to look at the situation at Self-control' $=0$ (which is the same as when Self-control $=m+s$, or the high level of Self-control).

To see if the effect of Touch-food at "low" levels of Self-control is significant, in her data, the researcher should replace Self-control by Self-control' $=$ Self-control $-m+s$. Then she should estimate equation (2). If $c$ is significant, then Touch-food significantly impacts Consumption at low levels of Self-control. In this case, when Self-control' $=0$, Self-control $=m-s$ or the low level of Self-control (1 standard deviation below the mean).

One thing researchers need to realize is that if $c$ is significant in equation (1), it indicates that Touch-food significantly affects consumption when Self-control $=0$. This is clearly not very insightful for the researcher since Self-control with a value of zero has little meaning, especially when it is measured on a 1-7 scale or a 1-100 scale. Even if Self-control is measured on a 0-100 scale, one needs to understand that $c$ being significant in equation (1) implies that Touch-food significantly affects consumption at the level of Self-control $=0$. Note that if the data is mean centered, the researcher will not deduct the mean from Self-control in computing self-control'. Also, self-control $=0$ will reflect mean Self-control.

Question 3: Spotlight analysis done at chosen values of the continuous moderator. What should a researcher do to run the spotlight analysis correctly if she estimates equation (1) and finds a significant interaction between Self-control and Touch-food (i.e., $d$ is significant)? However, instead of doing a spotlight analysis at one standard deviation above the mean as in question 1, she wants to look at the effect of Touch-food at a specific value of Self-control. Let's assume that Self-control is a measured variable with (continuous) values from 1 to 7 with a mean of 3 . She wants to focus on Self-control $=6$.

Option 1: In the data, she replaces Self-control by Self-control' $=$ Self-control +6

Option 2: In the data, she replaces Self-control by

Self-control' $=$ Self-control -6

Option 3: In the data, she replaces Self-control by

Self-control ${ }^{\prime}=$ Self-control $-3+6$

Option 4: In the data, she replaces Self-control by

Self-control ${ }^{\prime}=$ Self-control $-3-6$

Option 5: Other

Answer for Question 3: The logic for Q3 is very similar to that for Q1. In her data, she should (a) replace Self-control by Self-control' $=$ Self-control -6 (Option 2). Then (b) she should estimate equation (2).

Again, the correct answer of option 2 may seem counterintuitive and puzzling - why are we "subtracting" 6 from Self-control to focus on Self-control $=6$ ? Remember that when Self-control ${ }^{\prime}=0$, then equation (2) reduces to Consumption $=a+c$ Touch-food. If $c$ is significant in this equation, then it implies that Touch-food affects Consumption when Self-control ${ }^{\prime}=0$. However, when Self-control ${ }^{\prime}=0$, the original raw score for Self-control $=6$, which is what we want.

This is, in fact, the same logic as discussed in the "magic number zero" by Spiller et al. (2013). The counterintuitive nature of this magic number is highlighted here and explained in a different way.

Question 4: The puzzling nature of mean shifts (continuous $I V$ and categorical moderator). The final question is, What should a researcher do to run the spotlight analysis correctly if she estimates equation (1) and finds a significant interaction between Self-control and Touch-food (i.e., $d$ is significant), and wants to examine the effect of Self-control at Touch-food (yes)? Now we are treating the continuous variable Self-control as the independent variable and the categorical variable Touch-food as the moderator. In this case, since the moderator is not continuous, we do not really need a spotlight analysis; we can just do simple effect tests looking at the effect of Self-control on Consumption within each level of Touch-food. However, note that this simple effect test would be the same as doing a spotlight analysis focusing on the effect of self-control when one can touch the food. How should she do this spotlight?

Option 1: In the data, she replaces the value of Touch-food by Touch-food', where Touch-food' $=1$ when Touch-food is present and Touch-food ${ }^{\prime}=0$ when Touch-food is absent. Option 2: In the data, she replaces the value of Touch-food by Touch-food', where Touch-food' $=0$ when Touch-food is present and Touch-food ${ }^{\prime}=1$ when Touch-food is absent.

Answer for Question 4: (a) In her data, the researcher should replace the value of Touch-food by Touch-food', where Touch-food ${ }^{\prime}=0$ when Touch-food is present and Touch-food ${ }^{\prime}=1$ when Touch-food is absent (Option 2). Then (b) she should estimate the following equation:

$$
\begin{aligned}
\text { Consumption }= & a+b \text { Self }- \text { control }+c \text { Touch }- \text { food }^{\prime} \\
& +d\left(\text { Self }- \text { control } \times \text { Touch }- \text { food }^{\prime}\right)
\end{aligned}
$$

and look at the significance of $b$.

Dummy variables like Touch-food here are especially puzzling to handle in spotlight analysis. Typically, in a regression equation, and as we have in equation (1), when one can Touch-food, Touch-food takes the value of 1 . Thus, why are we changing things so that Touch-food ${ }^{\prime}=0$ when one can Touch-food? Note again that when Touch-food ${ }^{\prime}=0$, then equation (2) reduces to Consumption $=a+b$ Self-control. If $c$ is significant in this equation, then it implies that Self-control affects Consumption when Touch-food ${ }^{\prime}=0$. However, remember that when Touch-food ${ }^{\prime}=0$, then one can Touch-food, 
which is what we want. [The analysis for Touch-food $=0$ can be worked out similarly by the interested reader.]

When I have posed these questions to various groups, the most common response for Q1 is option 3, followed by option 1; for Q2, it is option 3; and for Q4, it is option 1. I have not posed Q3 to any group. I hope that with the tutorial and with this commentary, people will get the answer to Q3 right.

\section{Conducting spotlight analysis-some common contexts}

In looking at some common contexts for spotlight analyses, we first consider regression-based spotlight analyses and then their alternate approaches.

\section{Regression-based spotlight analyses}

Regression Case 1: binary categorical IV and continuous moderator. This context was discussed under Questions 1 and 2 in the previous section.

Regression Case 2: continuous IV and binary categorical moderator. This context was discussed under Question 4 in the previous section.

Regression Case 3: continuous IV and continuous moderator. Assume that Touch-food is a continuous independent variable and Self-control is a continuous moderator and we get a significant interaction between them. We want to see if Touch-food has a significant effect on Consumption at high values of Self-control. This case would be identical to Context 1 except that Touch-food would be a continuous variable. The researcher would still estimate equation (2) and see if $c$ is significant.

Regression Case 4: categorical IV and binary categorical moderator. Again, remember that spotlight analyses are needed when the moderator is a continuous variable. With two (manipulated) categorical variables, we can just use simple effect tests.

However, if one wants to conduct spotlight analysis instead of running simple effect tests, one can use the same logic that we have been following thus far. Let's assume that Self-control is not a measured continuous variable but is a manipulated categorical variable with two values (high and low) and Touch-food is again a categorical variable $(1=$ yes $/ 0=$ no $)$ and their interaction is significant so that $d$ is significant in equation (1) (reproduced below), but Self-control is now a dummy variable with Self-control $=1$ denoting high Self-control and Self-control $=0$ denoting low Self-control.

$$
\begin{aligned}
\text { Consumption }= & a+b \text { Self }- \text { control }+ \text { cTouch }- \text { food } \\
& +d(\text { Self }- \text { control } \times \text { Touch }- \text { food })
\end{aligned}
$$

Regression Case 4a: The effect of Self-control when one can touch the food. (a) Similar to Question 4, in the data, replace the value of Touch-food by Touch-food ${ }^{\prime}$, where Touch-food' $=0$ when Touch-food is present and Touch-food' $=1$ when Touch-food is absent. (b) Then estimate the equation (3)

$$
\begin{aligned}
\text { Consumption }= & a+b \text { Self }- \text { control }+ \text { cTouch }- \text { food }^{\prime} \\
& +d\left(\text { Self }- \text { control } \times \text { Touch }- \text { food }^{\prime}\right)
\end{aligned}
$$

and look at the significance of $b$. Remember that dummy variables like Touch-food here are especially puzzling to handle in spotlight analysis. To understand the conundrum, refer to the discussion under Question 4 in the previous section. And, importantly, please remember to report how you coded the categorical moderator.

Regression Case 4b: The effect of Touch-food when Self-control is high. In the data, replace the value of Self-control by Self-control', where Self-control' $=0$ for high Self-control and Self-control' $=1$ for low Self-control. (b) Then estimate equation (2) (reproduced as follows):

$$
\begin{aligned}
\text { Consumption }= & a+b \text { Self }- \text { control }^{\prime}+c \text { Touch }- \text { food } \\
& +d\left(\text { Self }- \text { control }^{\prime} \times \text { Touch }- \text { food }\right)
\end{aligned}
$$

and look at the significance of $c$. If puzzled, refer to the discussion under Question 4 in the previous section. Again, please remember to report how you coded the categorical moderator.

Regression Case 5: Trinary categorical independent variable and continuous moderator. Another spotlight analysis situation where I have seen consumer psychologists get puzzled is when the categorical independent variable has three levels (say, the food cannot be touched, can be touched, and can be picked up). In this situation, researchers often do not know where to start. In fact, a spotlight is quite easy to do in this situation. Using two dummies to represent the three levels of Touch-food (D_touch $=1$ and D_pickup $=0$ for touching the food; D_touch $=0$ and D_pickup $=1$ for picking up the food; D_touch $=0$ and D_pickup $=0$ for not touching the food), one would first estimate as follows:

$$
\begin{aligned}
\text { Consumption }= & a+b \text { D_touch }+ \text { cD_pickup }+\mathrm{dSelf} \\
& - \text { control }+ \text { eD_touchñSelf }- \text { control } \\
& + \text { fD_pickupñSelf }- \text { control }
\end{aligned}
$$

Depending on which interaction was significant, one would probe it further using spotlight analyses similar to the one for a binary categorical variable that we discuss in Question 1 and Question 2. For instance, if coefficient $e$ was significant and one wanted to check if touching the food (versus not) was significant at high levels of Self-control, then one would need to mean shift the data to reflect high levels of Self-control (refer to Question 1 for how to do this correctly). Then one would need to look at the significance of $b$ in the estimated regression of the mean shifted data (refer to Question 2). [If one wants to look at the effect of touching versus picking-up for different levels of self-control, one would modify the choice of dummies so that D_touch $=1$ and D_notouch $=0$ for touching the food; D_touch $=0$ and D_notouch $=1$ for not touching the food; D_touch $=0$ and D_notouch $=0$ for picking up the food.]

Regression Case 6: spotlight at focal points. Upon consideration, one can see that doing a spotlight analysis at one standard deviation above and below the mean is just another approach, albeit a less problematic one than the median split of the continuous moderator that researchers were doing earlier. However, there are other approaches, besides the traditional spotlight analysis, as well. 
One such approach is to do spotlight analysis at different chosen points (focal points) and has been discussed also by Irwin and McClelland (2001) and recently by Spiller et al. (2013). This is also the spotlight analysis discussed under Question 3. Note that the "focal point" approach is sometimes called the "absolute point" approach.

To repeat from Question 3 posed earlier, the researcher may estimate equation (1) and find a significant interaction between Self-control and Touch-food (i.e., $d$ is significant). Now, she may want to look at the effect of Touch-food at specific values of Self-control. Let's assume that Self-control is a measured variable with (continuous) values from 1 to 7 with a mean of 3. She may want to focus on Self-control at values of 2,4 , and 6 . If she wants to do this, she should run three new analyses. In these three analyses, in her data, she should replace self-control with Self-control' ${ }^{\prime}$ where Self-control' ${ }^{\prime}=$ Self-control - 2, Self-control - 4, and Self-control - 6, respectively. For each of these three new data sets, she should then estimate equation (2).

Fig. 2 displays the effect of Touch-food on consumption when Self-control varies between 1 and 7 .

Regression Case 7: three-way interaction. Sometimes, we may have a significant three-way interaction between the independent variable (e.g., Touch-food) and two moderators (e.g., Self-control and Need-for-touch). We obtain this significant interaction in the following equation:

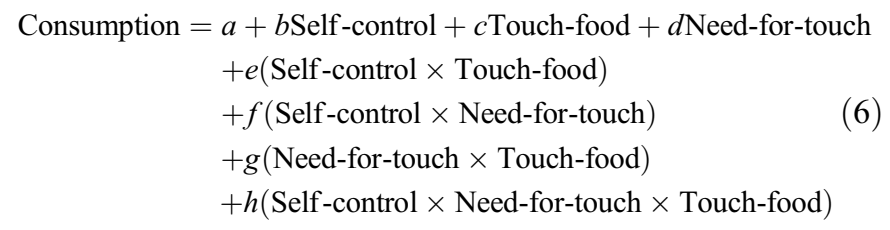

Now, we want to test if Touch-food has an effect on consumption if consumers are high in Need-for-touch but have low Self-control. That is, we want to direct a spotlight on high Need-for-touch with low Self-control. How do we do this?

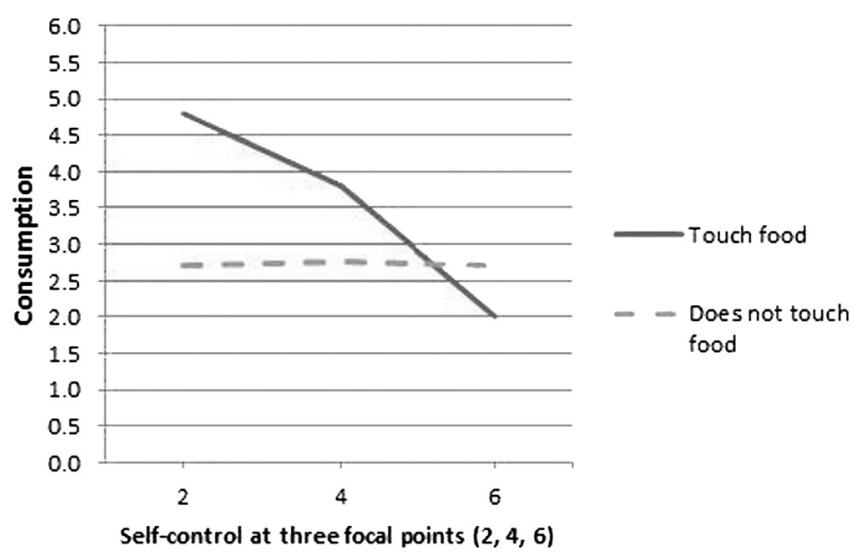

Fig. 2. Hypothetical effect of self-control and touch-food (yes/not) on consumption (self-control at focal points).
First, in the data, we replace Self-control with the following: Self-control' $=$ Self-control $-m_{\mathrm{sc}}+s_{\mathrm{sc}}$, where $m_{\mathrm{sc}}$ is the mean of Self-control and $s_{\mathrm{sc}}$ is its standard deviation.

We also replace in the data Need-for-touch with the following:

Need-for-touch ${ }^{\prime}=$ Need-for-touch $-m_{\mathrm{nft}}-s_{\mathrm{nft}}$, where $m_{\mathrm{nft}}$ is the mean of Need-for-touch and $s_{\mathrm{nft}}$ is its standard deviation.

Next, in equation (6), we replace all instances of Self-control with Self-control' and all instances of Need-for-touch with Need-for-touch' ${ }^{\prime}$ and estimate the new equation. In this estimated equation, we see if $c$ is significant.

Fig. 3 shows the hypothetical three-way interaction between Touch-food with Self-control and Need-for-touch on Consumption. Fig. $3 \mathrm{a}$ and $\mathrm{b}$ display the effect of Self-control and Touching Food on Consumption when consumers are high or low in Need-for-touch, respectively.

The seven spotlight analysis cases we considered were predicated on using regression. We now consider alternate (to regression) approaches for doing spotlight analysis, specifically PROCESS.

\section{Alternate approaches for spotlight: some analyses covered by process}

While one can use regression to conduct spotlight analyses, there are also free modeling tools for SPSS and SAS (in many instances) to do so. The most commonly used free tools are covered in the software PROCESS by Hayes [available at http://afhayes.com/introduction-to-mediationmoderation-and-conditional-process-analysis.html; documentation covered in Hayes (2013), but Hayes calls it "conditional effects" or "simple slopes" (not "spotlight analysis")].

PROCESS Case 1: default case-traditional spotlight analysis. The default case in PROCESS computes conditional effects (or does spotlight analyses) at the mean, mean plus 1 standard deviation, and mean minus one standard deviation of the moderator variable. These are considered low, medium, and high values of the moderator. The programming command for this is as follows:

$\begin{aligned} \text { Process vars } & =\mathrm{IV} \text { moderator } \mathrm{DV} / y=\mathrm{DV} / x=\mathrm{IV} / m \\ & =\text { moderator } / \operatorname{model}=1 .\end{aligned}$

In the example we considered, Consumption would be the DV, Touch-food (yes/no) the IV and Self-control the moderator.

However, we may want other values for the low, medium and high values of the moderator-e.g., 25th, 50th, and 75th percentile. We may desire spotlight analyses at more than three values of the moderator. Alternately, we may want additional understanding of where (in its range) the moderator significantly impacts the independent variable's effect on the dependent variable and where it does not. For these possibilities, there are (at least) three alternate approaches all of which can be perused in greater detail in Hayes (2013).

PROCESS Case 2: default quantiles: If one adds the subcommand of "quantile $=1$ " in the PROCESS command 
a) Consumers with high levels of Need -for-Touch (1 standard deviation above the mean)

High Need-for-Touch

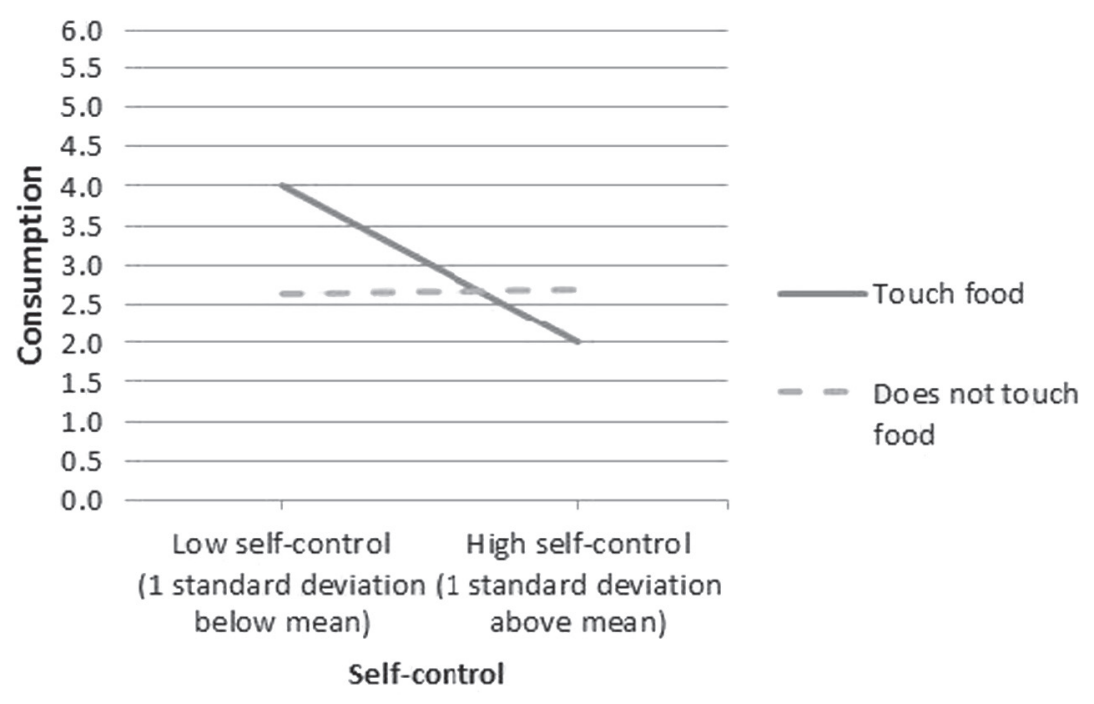

b) Consumers with low levels of Need -for-Touch (1 standard deviation below the mean)

\section{Low Need-for-Touch}

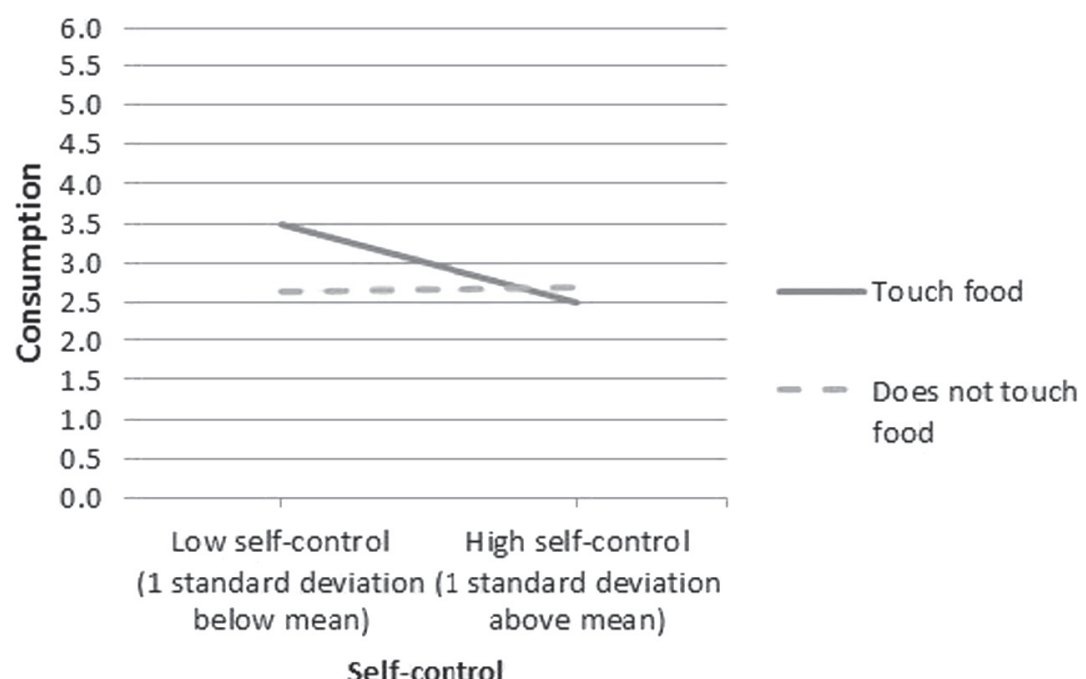

Fig. 3. Hypothetical effect of self-control and touch-food (yes/not) and need-for-touch on consumption (three-way interaction). (a) Consumers with high levels of Need-for-touch (1 standard deviation above the mean). (b) Consumers with low levels of Need-for-touch (1 standard deviation below the mean).

provided earlier, then the macro does spotlight analyses at the 10th, 25th, 50th, 75th, and 90th percentiles of the moderator:

Process vars $=\mathrm{IV}$ moderator DV $/ y=\mathrm{DV} / x=\mathrm{IV} / m$

$$
=\text { moderator } / \text { model }=1 / \text { quantile }=1 \text {. }
$$

Suppose in the example we considered earlier, Touch-food (yes/no as IV) interacts with Self-control (m, the moderator) to affect Consumption (DV). [If one wants to use regression instead of PROCESS, we would perform spotlights for focal points of Self-control $=10$ th, 25th, 50th, 75th and 90th percentiles of the moderator. These values would be determined first before running the regressions given under Question 3].
PROCESS Case 3: focal point approach (or pick an absolute point approach) - This approach is useful for proactively choosing values of the moderator where the researcher thinks the moderator will and will not impact the effect of the independent variable on the dependent variable. In this way, any turning points (from significance to non-significance) may be found. This method has been discussed by several scholars (e.g., Bauer \& Curran, 2005; also see page 7 of afhayes.com/public/ process.pdf). For instance, we may expect that the independent variable has a significant impact on the dependent variable when the moderator's (absolute) value is V1 but not when it is V2. In that case, one may want to do a spotlight at V1 and V2. 
For this, one can run:

$$
\begin{aligned}
\text { Process vars } & =\mathrm{IV} \text { moderator } \mathrm{DV} / y=\mathrm{DV} / x=\mathrm{IV} / m \\
& =\text { moderator } / \text { model }=1 / \mathrm{MMODVAL}=\mathrm{V} 1 .
\end{aligned}
$$

Process vars $=\mathrm{IV}$ moderator $\mathrm{DV} / y=\mathrm{DV} / x=\mathrm{IV} / m$

$$
=\text { moderator } / \text { model }=1 / \text { MMODVAL }=\mathrm{V} 2 \text {. }
$$

[If one wants to use regression instead of PROCESS, we would perform spotlights for focal points of Self-control $=\mathrm{V} 1$ and V2 as discussed under Question 3. For instance, in Fig. 2, Touch-food significantly affects consumption at Self-control $=4$ but not at Self-control $=6$. There is a turning point from significance to non-significance in between Self-control values of 4 and 6.].

PROCESS Case 4: Johnson-Neyman approach. When the moderator is continuous, one can look for the turning points for where exactly, in the absolute value of the moderator, the effect of the independent variable turns from non-significance to significance (for a pre-specified significance level). This is done using the Johnson-Neyman technique (Bauer \& Curran, 2005; Hayes, 2013; Hayes \& Matthes, 2009).

For this approach, use:

$$
\begin{aligned}
\text { Process vars } & =\mathrm{IV} \text { moderator } \mathrm{DV} / y=\mathrm{DV} / x=\mathrm{IV} / m \\
& =\text { moderator } / \text { model }=1 / \text { quantile }=1 / \mathrm{JN}=1 .
\end{aligned}
$$

[If one is running regressions instead of using PROCESS, one can do this with a systematic search through focal points to arrive at the turning point.]

PROCESS Case 5: moderated mediation. PROCESS also allows for testing a continuous moderator along with a mediator. If we have hypothesized a moderated mediation where the moderator affects the relationship (path) between the independent variable and the mediator, then the code is:

$$
\begin{aligned}
\text { Process vars } & =\mathrm{IV} \text { mediator } \mathrm{DV} \text { moderator } / y=\mathrm{DV} / x=\mathrm{IV} / m \\
& =\text { mediator } / w=\text { moderator } / \text { model }=7 / \text { quantile } \\
& =1 .
\end{aligned}
$$

If, instead, we hypothesize that the moderation happens after the mediation, i.e., the moderator affects the relationship (path) between the mediator and the dependent variable, we should use the following:

$$
\begin{aligned}
\text { Process vars } & =\mathrm{IV} \text { mediator } \mathrm{DV} \text { moderator } / y=\mathrm{DV} / x \\
& =\mathrm{IV} / m=\text { mediator } / v=\text { moderator } / \text { model } \\
& =14 / \text { quantile }=1 .
\end{aligned}
$$

Note that in all the PROCESS code we have offered so far, there is a model number included. Hayes (2013) includes numerous possible models and codes for testing them. In the moderated mediation models just discussed, the models are 7 (path from independent variable to mediator is moderated) and 14 (path from mediator to dependent variable is moderated). These two models are used for used for ease of explanation, and they assume that there is no moderation of the direct effect of $X$ on $Y$. If the moderator is hypothesized to affect both the indirect path between $X$ and $M$ and the direct path from $X$ to $Y$, one should use model 8 instead of model 7, and model 15 instead of model 14. The syntax remains the same, except for the change in model number. Of course, the reporting of results changes to include discussion of the (possible) moderation of the direct path from $X$ to $Y$.

These are just five alternatives to the standard spotlight analysis. Other approaches also exist. However, these five are likely to be the most commonly used ones. We now turn our attention to reporting of spotlight analyses.

\section{Reporting spotlight analysis and the Johnson-Neyman technique}

How opaque are our mistakes? It would be interesting to know how many readers who have used spotlight analyses in their papers answered the four questions correctly. The unfortunate thing is that with the way people report the data (following Fitzsimons, 2008), reviewers and readers have no way of knowing if the authors made a mistake. In the standard way of reporting data, no details are given for exactly how the spotlight analysis was done. The authors merely report that

"A spotlight analysis at one standard deviation above the mean of Self-control showed a significant difference such that high Self-control consumers consumed less when no Touch-food was present versus when a Touch-food was present."

Authors could, in fact, have chosen any of the first four options (options 1-4) in Q1 to conduct the spotlight analysis (three of which are wrong). One can only hope that they chose the correct option.

More detailed reporting and some templates. I hope that this article will reduce future mistakes. Also, I hope that authors will be asked to report how exactly a spotlight was done. For instance, when doing the spotlight analysis, the authors can say,

"To conduct a spotlight analysis at one standard deviation above the mean of Self-control (i.e., at high Self-control), we first mean-shifted the data. In order to do this, we modified the original Self-control data by subtracting from it the mean and one standard deviation. We then conducted our analysis on the mean-shifted data. The spotlight analysis showed that ... The equations we used for mean shifting the data are provided below..." [the equations could also be provided in a web appendix. If the authors dummy coded any data, then they should also note "Variable () was coded as ..."]

If the authors use focal point values for their spotlight analyses, they should report,

"To conduct a spotlight analysis at Self-control $=6$, we first mean-shifted the data. In order to do this, we modified the original Self-control data by subtracting 6 from it. We then conducted our analysis on the mean-shifted data. The spotlight analysis showed that ... The equations we used for mean shifting the data are provided below..." [the equations could also be provided in a web appendix. 
If the authors dummy coded any data, then they should also note "Variable () was coded as ..."]

If the authors have a three-way interaction, they can say,

"We obtained a significant three-way interaction between Touch-food, Self-control, and Need-for-touch on Consumption. To point a spotlight at the effect of Touch-food on Consumption for people high on Need-for-touch with low Self-control, we mean shifted the data. In order to do this, we modified the original Need-for-touch data by subtracting from it the mean and one standard deviation. We also modified the original Self-control data by subtracting from it the mean but adding one standard deviation. We then conducted our analysis on the mean-shifted data. The equations we used for mean shifting the data are provided below..." [the equations could also be provided in a web appendix. If the authors dummy coded any data, then they should also note "Variable () was coded as ...”]

If the authors use Johnson-Neyman, they could report, for instance,

"Since the moderator (Self-control) was continuous, we looked for the turning points for where exactly, in the absolute value of the moderator, the effect of the independent variable (Touchfood: present versus absent) turns from non-significance to significance (for a pre-specified significance level of 0.05). This is done using the Johnson-Neyman technique (Bauer \& Curran, 2005; Hayes, 2013; Hayes \& Matthes, 2009).

For this approach, we used the command:

[Process vars $=$ Touch-food Self-control Consumption $/ y=$ Consumption $/ x=$ Touch-food $/ m=$ Self-control $/$ model $=1 / \mathrm{JN}=1$.]

The Johnson-Neyman technique showed that Self-control at a value of 5 is the turning point from non-significance to significance of the effect of Touch-food (present versus absent). The presence of a Touch-food is associated with significantly greater Consumption than the absence of a Touch-food for values of self-control below 5. When Selfcontrol is greater than 5 , then while the presence (versus absence) of a Touch-food is associated with greater Consumption, the difference between Consumption at Touchfood-present and Touch-food-absent is not significant...”

Lastly, if the authors have a moderated mediation, ${ }^{1}$ they can state,

"We hypothesized that Imagery mediated the effect of Touchfood (X) on Consumption (Y). Furthermore, we hypothesized

\footnotetext{
${ }^{1}$ Hayes (2013) notes how the way mediated moderation model is estimated is mathematically identical to moderated mediation (when the moderator operates on $\mathrm{X} \rightarrow \mathrm{Med}$ ). I agree with Hayes preferring the use of moderated mediation over mediated moderation. This takes the focus off interpreting the interaction ( $\left.\mathrm{X}^{*} \mathrm{Mod}\right)$ as the causal agent and shifts it back to the independent variable (X).
}

a significant moderating effect of Self-control (Mod) on the relationship (or path) between Touch-food (X) and Imagery (Med). We tested for this moderated mediation model using Hayes (2013) PROCESS macro for Model 7 with 5,000 bootstrapped samples (Hayes, 2013). We used the command:

[Process vars $=$ Touch-food imagery Consumption Selfcontrol $/ y=$ Consumption $\quad / x=$ Touch-food $/ m=$ Imagery $/$ $w=$ Self-control $/$ model $=7 /$ boot $=5000 /$ quantile $=1$.]

PROCESS results showed evidence for a significant moderating effect of Self-control (Mod) on the relationship (or path) between Touch-food $(X)$ and Imagery (Med) $(B=\ldots ; t=\ldots ; p=. \ldots)$. Additionally, controlling for Touch-food $(X)$, Imagery (Med) had a significant effect on Consumption $(M \rightarrow Y ; B=\ldots ; t=\ldots ; p=. \ldots)$; controlling for Imagery (Med), the direct effect of Touchfood $(X)$ on Consumption $(Y)$ was not significant $(B=$ _; $t=\ldots ; p=$._. . PROCESS also allowed for further probing of the moderating effect of Self-control on the Touch-food (X)-Imagery (Med) relationship. Specifical$l y$, it allowed for inferential tests of the effect at the quantiles. We found evidence for a significant indirect effect of Touch-food on Consumption at the higher levels of Self-control $\left(50^{\text {th }}, 75^{\text {th }}\right.$ and $90^{\text {th }}$ percentile of Selfcontrol)- $95 \%$ confidence intervals for the three tests lay between _ and _,_ and _, and _ and _, respectively, implying that none of the three intervals included zero. We did not obtain a significant effect for lower levels of Self-control (10th and 25th percentiles of Self-control)."

Note that here we have adopted model 7 for moderated mediation. Clearly, the researcher should check if other models are more appropriate-e.g., check if there are other moderating effects which would require a different model. Note also that Hayes (2013) remarks how the way mediated moderation model is estimated is mathematically identical to moderated mediation (when the moderator operates on $\mathrm{X} \rightarrow$ Med). I agree with Hayes preferring the use of moderated mediation over mediated moderation. This takes the focus off interpreting the interaction $\left(\mathrm{X}^{*} \mathrm{Mod}\right)$ as the causal agent and shifts it back to the independent variable (X).

Figures for spotlight analyses. In reporting the results of spotlight analyses, figures can help the reader get a better grasp on the data. Figs. 1 to 3 are created by using the estimated regression equations. For instance, in Fig. 1, one needs 4 points to plot the 2 lines - consumption for high (low) touch at high (low) self-control. One uses the estimated equation (1) to get estimated consumption amounts at these four data points. Fig. 2 is made similarly using equation (2) and Fig. 3 by using equation (6). Fig. 4 is made similarly to Fig. 3 but using specific percentiles. Note that if journals have a word limit, perhaps some of the details and figures could be moved to a web appendix.

Note that the syntax for the Johnson-Neyman technique needs to include subcommand "plot $=1$ " to create a figure. This subcommand generates a table of predicted values of 


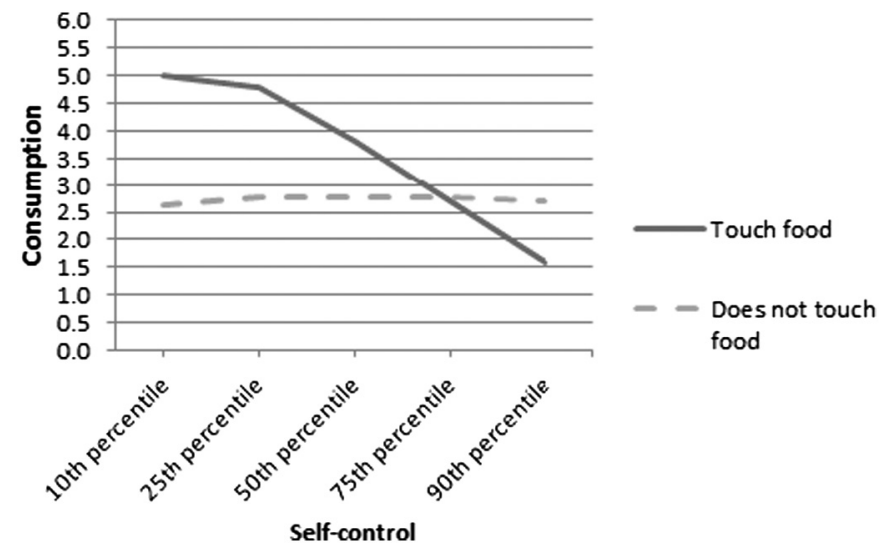

Fig. 4. Hypothetical effect of consumer Self-control and Touch-food on consumption (spotlight at percentiles).

$\mathrm{Y}$ from the model using various values of $\mathrm{X}$, and of the moderator.

\section{Conclusion}

I hope that this succinct article will help researchers conduct spotlight analysis with ease and do it accurately. Second, I hope that editors and reviewers will urge authors to report how exactly they did their spotlight analysis. After all, spotlight analysis replaced the practice of dichotomizing continuous variables partly because the practice could produce spurious results. This additional effort by journals to ensure that spotlight is done accurately will also decrease the likelihood of spurious results being provided using spotlight analysis. Lastly, I hope authors will use alternate approaches to the traditional analysis done at one standard deviation above and below the mean value of the moderator - for example, quantiles and focal points, and look for turning points in the significance of effects. It is time we focused a spotlight on the way we have been conducting spotlight analysis!

\section{References}

Aiken, L. S., \& West, S. G. (1991). Multiple regression: Testing and interpreting interactions. Newbury park, CA: Sage Publications.

Bauer, D. J., \& Curran, P. J. (2005). Probing interactions in fixed and multilevel regression: Inferential and graphical techniques. Multivariable Behavioral Research, 40, 373-400.

Cohen, J. (1983). The cost of dichotomization. Applied Psychological Measurement, 7, 249-253.

Fitzsimons, G. J. (2008). Death to dichotomizing. Journal of Consumer Research, 35(1), 5-8 (Editorial).

Hayes, A. F. (2013). Introduction to mediation, moderation, and conditional process analysis: A regression based approach. The Guilford Press.

Hayes, A. F., \& Matthes, J. (2009). Computational procedures for probing interactions in OLS and logistic regression: SPSS and SAS implementations. Behavioral Research Methods, 41, 924-936.

Iacobucci, D., Posovac, S. S., Kardes, S. R., Schneider, M. J., \& Popovich, D. L. (2015a). Toward a more nuanced understanding of the statistical properties of a median split. Journal of Consumer Psychology, 25(4), 662-665.

Iacobucci, D., Posovac, S. S., Kardes, S. R., Schneider, M. J., \& Popovich, D. L. (2015b). The median split: Robust, refined and revived. Journal of Consumer Psychology, 25(4), 690-704.

Irwin, J. R., \& McClelland, G. H. (2001). Misleading heuristics and moderated multiple regression models. Journal of Marketing Research, 38(February), 100-109.

Irwin, J. R., \& McClelland, G. H. (2003). Negative consequences of dichotomizing continuous predictor variables. Journal of Marketing Research, 40(August), 366-371.

Jaccard, J., Turrisi, R., \& Wan, C. K. (1990). Interaction effects in multiple regression. Newbury Park, CA: Sage Publications.

MacCallum, R. C., Zhang, S., Preacher, K. J., \& Rucker, D. D. (2002). On the practice of dichotomization of quantitative variables. Psychological Methods, 7, 19-40.

McClelland, G. H., Lynch, J. G., Irwin, J. R., Spiller, S. A., \& Fitzsimons, G. J. (2015). Median splits, type II errors, and false-positive consumer psychology: Don't fight the power. Journal of Consumer Psychology, 25(4), 679-689.

Pham, M. T. (2015). Is it okay to dichotomize: A research dialog. Journal of Consumer Psychology, 25(4), 650-651.

Rucker, D. D., McShane, B. B., \& Preacher, K. J. (2015). A researcher's guide to regression, discretization, and median splits of continuous variables. Journal of Consumer Psychology, 25(4), 666-678.

Spiller, S. A., Fitzsimons, G. J., Lynch, J. G., Jr., \& McClelland, G. H. (2013). Spotlights, floodlights, and the magic number zero: Simple effects tests in moderated regression. Journal of Marketing Research, 50(2), 277-288. 